\title{
ZPesearchsoure $\begin{array}{ll}\text { Research Square } & \text { They should not be considered conclusive, used to inform clinical practice, } \\ \text { or referenced by the media as validated information. }\end{array}$
}

\section{Renal Oxygenation in male Metabolic Syndrome as evaluated by Blood Oxygen Level-Dependent Magnetic Resonance Imaging}

\section{Yongqiang Li ( $\sim$ liyongqiang851@163.com )}

The Third Affiliated Hospital of Southern Medical University

\section{Xiaodong Zhang}

The Third Affiliated Hospital Of Southern Medical University

Chijian Li

The Third Affiliated Hosital Of Suthern Medical University

Shisi Li

The Third Affiliated Hospital Of Southern Medical University

\section{Shiyi Liang}

The Third Affiliated Hospital Of Southern Medical University

\section{YiHao Guo}

Southern Medical University Nanfang Hospital

\section{Ge Qian}

The Third Affiliated Hospital Of Southern Medical University

\section{Yuxiang Huang}

The Third Affiliated Hospital of Southern Medical University

\section{Weicheng Xv}

The Third Affiliated Hospital Of Southern Medical University

\section{Research}

Keywords: renal oxygenation, metabolic syndrome, BOLD-MRI

Posted Date: July 23rd, 2020

DOl: https://doi.org/10.21203/rs.3.rs-42697/v1

License: (c) (1) This work is licensed under a Creative Commons Attribution 4.0 International License. Read Full License 


\section{Abstract}

Background- Blood Oxygen Level-Dependent Magnetic Resonance Imaging (BOLD-MRI) provide regional measurements of oxygen content using deoxyhemoglobin paramagnetic characteristics. Metabolic Syndrome (MS) could affect renal oxygenation, which have an impact on the clinical course of the disease. The goal of this study was to evaluate BOLD-MRI findings in kidneys of male patients with MS.

Materials and Methods- A total of 24 males patients with MS were collected in the hospital and 24 male volunteers were selected as the control group. All volunteers underwent a renal scan using 3.0T magnet (Philips, Achieva). $\mathrm{R}_{2}{ }^{*}$ of the renal cortex and medulla was measured on BOLD images.

Results-Medullary $\mathrm{R}_{2}{ }^{*}\left(\mathrm{MR}_{2}{ }^{*}\right)$ and Medulla/Cortex $\mathrm{R}_{2}{ }^{*}$ ratioهMCR】 were significantly lower in MS patients $\left(\mathrm{MR}_{2}{ }^{*}=24.39 \pm 2.18 \mathrm{~s}^{-1}, \mathrm{MCR}=1.29 \pm 0.14\right)$ than in controls $\left(\mathrm{MR}_{2}{ }^{*}=28.21 \pm 4.70 \mathrm{~s}^{-1}, \mathrm{MCR}=1.53 \pm 0.28\right)$ $(P<0.01)$, and cortical $R_{2}{ }^{*}\left(C_{2}{ }^{*}\right)$ values were not significantly different between the two group.

Conclusions- The $\mathrm{MR}_{2}{ }^{*}$ and MCR were lower in male patients with MS compared to controls. $\mathrm{MR}_{2}{ }^{*}$ and MCR were potential imaging biomarkers for prediction of early renal impairment in male MS.

\section{Background}

Metabolic Syndrome (MS) and chronic kidney diseases $₫$ CKD $\bigotimes$ have become global health problems and increasing worldwide. Data from National Health and Nutrition Examination Survey (NHANES) showed that overall prevalence of the metabolic syndrome in the United States was 33\% from 2003 to $2012^{[1]}$ and the prevalence of CKD was $13.1 \%$ in the United States from 1999 to $2004^{[2]}$. MS was a significant risk factor for CKD囚but the pathogenesis is not yet well understood ${ }^{[3-5]}$.

There are increasing evidences suggesting an association between CKD and chronic renal hypoxia ${ }^{[6,7]}$. In order to understand the renal oxygenation status in MS, it is necessary to monitor renal oxygenation in these patients noninvasively.

BOLD-MRI is currently the only noninvasive technique that evaluate oxygenation in vivo tissue. BOLDMRI is sensitive to magnetic field inhomogeneity induced by deoxyhemoglobin. As field inhomogeneity makes water molecules de-phase faster, the acquired gradient-echo signals decay faster with echo time. With different echo time values, we apply an exponential decay function to calculate parameter $R_{2}{ }^{*}\left(R_{2}{ }^{*}=\right.$ $\left.1 / T_{2}{ }^{*}\right)$. BOLD-MRI is based on the assumption that blood oxygen level is in equilibrium with that in tissue. In the interest of simplicity, we ascribed the changes in $\mathrm{R}_{2}{ }^{*}$ values to changes in oxygenation, suggesting that an increase in $\mathrm{R}_{2}{ }^{*}$ values implies reduced oxygenation ${ }^{[8-10]}$. BOLD-MRI has been used to provide regional measurements of oxygen content in experimental models and human kidney diseases ${ }^{[11-15]}$. However, none of the published studies examined renal oxygenation in MS patients. The purpose of the study was to evaluate BOLD-MRI findings in male patients with MS. 


\section{Materials And Method}

The protocol of this study was discussed, approved and recorded by the ethics committee of the hospital. 24 male patients with MS were enrolled in the study and 24 male volunteers were selected as the control group. Informed consent was obtained from all participants involved in this study.

\subsection{Determination of MS}

According to the definition of 2013 Chinese diabetes society $\llbracket C D S$ 2013) $\llbracket M S$ was defined as three or more of the following abnormalities: (1) waist circumference $\geq 90 \mathrm{~cm}$ for men, (2) Fasting Triglyceride $(T G) \geq 1.70 \mathrm{mmol} / \mathrm{L}$, (3) Fasting High density lipoprotein cholesterol (HDL-C) < $1.04 \mathrm{mmol} / \mathrm{L}$, (4) Blood pressure $\geq 130 / 85 \mathrm{mmHg}$ and/or receiving treatment for previously diagnosed hypertension, (5) Fasting blood glucose $(F B G) \geq 6.1 \mathrm{mmol} / \mathrm{L}$ or oral glucose tolerance test (OGTT) $2 \mathrm{~h}$ blood glucose $(\mathrm{BG}) \geq 7.8$ $\mathrm{mmol} / \mathrm{L}$ and/or confirmed diabetes that is under treatment ${ }^{[16]}$.

\subsection{The inclusion and exclusion criteria}

The inclusion criteria of MS were as follows: (1) age between 18-75 years old, (2) without contraindications of MRI examination, (3) eGFR is not less than $60 \mathrm{ml} / \mathrm{min} / 1.73 \mathrm{~m}^{2}$. (4) in accordance with the MS definition of CDS 2013. Exclusion criteria of MS were as follows: (1) combined with other kidney diseases such as polycystic kidney disease, glomerular nephritis, etc. (2) difficult to analyze because of poor image quality (3) nephrotoxic and vascular drugs were used within 8 weeks before the examination. The inclusion criteria of volunteers were as follows: (1) age between 18-75 years old. (2) without contraindications of MRI examination. (3) eGFR is normal. Exclusion criteria of volunteers were as follows: (1) difficult to analyze because of poor image quality. (2) Nephrotoxic and vascular drugs were used within 8 weeks before the examination.

\subsection{Physical measures}

Anthropometric indices including height, blood pressure, waist circumference and weight was measured by trained physicians and nurses. All subjects wear light clothing without shoes and height and weight were measured by an automated height and weight machine. Participants were in a standing position with arms on side, legs straight, and knees together, with feet flat pointed outward. Waist circumference was measured in a horizontal plane, midway between the inferior margin of the last rib and the superior margin of the iliac crest. Blood pressure was measured twice by trained nurse, and the average value of these 2 measuring points was recorded (before the blood pressure was measured, all participants need at least 5 minutes to rest).

\subsection{Laboratory Assays}


For all Participants, venous blood were collected after an overnight fast. Serum creatinine (Scr) was measured with an enzymatic method on an autoanalyzer (Hitachi 7170, Hitachi, Tokyo, Japan). The eGFR was obtained from the Modification of Diet in Renal Disease (MDRD) equations. eGFR ( $\mathrm{ml} / \mathrm{min} / 1.73 \mathrm{~m}^{2}$ ) $=175 \times(\mathrm{Scr})^{-1.234} \mathrm{X}(\mathrm{Age})^{-0.179}[17]$. Determination of High density lipoprotein (HDL) concentration was performed by commercially available reagents (Shanghai Gensource Co., Ltd, Shanghai, China). Total cholesterol (TC) and triglyceride (TG) levels were determined enzymatically with commercially available reagents (Roche Diagnostics, Mannheim, Germany). SUA level was measured with a colorimetric method (Roche Diagnostics, Mannheim, Germany). HOMA-IR was calculated according to the formula: HOMA-IR = [fasting plasma glucose $(\mathrm{mmol} / \mathrm{L}) \mathrm{X}$ fasting insulin $(\mathrm{mU} / \mathrm{L}) / 22.5]^{[18]}$.

\subsection{BOLD-MRI: Acquisition}

BOLD-MRI examinations were performed on a 3.0 T magnet (Philips, Achieva). The data were acquired by a breath-hold (15 second) multiple gradient echo sequences. Parameters were as followed: 16 echoes, echo time (TE): $1.20 \mathrm{~ms}$; repetition time (TR): $16 \mathrm{~ms}$; spacing: $0 \mathrm{~mm}$; thickness: $5 \mathrm{~mm}$; bandwidth: 1753.5 $\mathrm{Hz}$; flip angle: 25 degrees; field of view (FOV): 350 × 350 mm; matrix: 176 × 173. All the subjects were fasting 4 hours before MRI scan.

\subsection{BOLD-MRI: Analysis and data measurement}

The data were analyzed by ITK-SNAP software and $\mathrm{R}_{2}{ }^{*}$ values were measured and generated by the software automatically by regions of interest (ROI). Two coronal slices were acquired as measurement plane through the middle of the kidneys. ROI with fixed size were placed in the upper, middle and lower pole of both kidneys in cortex and medulla. Five ROIs were placed in cortex and medulla, respectively, avoiding the renal collection system, yielding a total of 20 ROls in both kidneys per slice. $\mathrm{R}_{2}{ }^{*}$ value were measured twice by two experienced radiologists and the final mean $\mathrm{R}_{2}{ }^{*}$ value was obtained by averaging values from two measurements. Because $R_{2}{ }^{*}$ values are easily affected by several external effects such as magnetic field inhomogeneity, coil positions, etc ${ }^{[19]}$, we also calculated the Medulla/Cortex $\mathrm{R}_{2}{ }^{*}$ ratio $\triangle M C R \otimes$ for each subject besides cortical $R_{2}{ }^{*}\left(\mathrm{CR}_{2}{ }^{*}\right)$ and Medullary $\mathrm{R}_{2}{ }^{*}\left(\mathrm{MR}_{2}{ }^{*}\right)$. Assuming that $\mathrm{CR}_{2}{ }^{*}$ and $\mathrm{MR}_{2}{ }^{*}$ are similarly influenced by the same external effects, the MCR will be less impacted by external effects.

\subsection{Statistical analyses}

All statistical analysis were performed by SPSS (IBM, Armonk, NY; statistics 20.0). Continuous variables were shown as mean \pm one standard deviation if data had normal distribution. Intraclass correlation coefficient (ICC) of two measurement data was calculated to evaluate interobserver consistency. If ICC values is more than 0.75 , a high reliability was considered and the data were used. To detect the different 
renal oxygenation between cortex and medulla, $\mathrm{R}_{2}{ }^{*}$ value was compared in each group by paired $\mathrm{t}$ test. To test the difference between MS group and control group, $\mathrm{CR}_{2}{ }^{*} \mathrm{\triangle MR}_{2}{ }^{*}$ and $\mathrm{MCR}$ were compared respectively (Independent samples t test). Besides, the relationship between $\mathrm{R}_{2}{ }^{*}$ values and eGFR was assessed by Pearson correlation analysis. $\mathrm{P}<0.05$ was considered as significant.

\section{Result}

\subsection{Clinical characteristics}

Baseline clinical characteristics of two groups were shown in Table 1. Participants of two groups were well matched for age $\$ height, serum creatitine (Scr), serum uric acid (SUA), total cholesterol (TC), low density lipoprotein (LDL) and hemoglobin (HGB). The weight, body mass index (BMI), blood pressure $(B P)$, waist circumference (WC), fasting insulin (FIN), fasting blood glucose (FBG), triglyceride (TG), alanine aminotransferase $\triangle A L T \rrbracket$, aspartate aminotransferase $\triangle A S T \rrbracket$ in MS group were significantly higher than those in control group and high density lipoproptein (HDL) and eGFR were significantly lower than those of control groups, respectively.

\subsection{BOLD-MRI image and Intraclass correlation coefficient (ICC)}

On the BOLD-MRI image, the demarcation of the renal cortex and medulla is clear. Intraclass correlation coefficient of two measurement data was calculated to evaluate interobserver consistency and ICC value of $\mathrm{R}_{2}{ }^{*}$ was $78.7 \%$ for cortex and $88.6 \%$ for medulla (both ICC $>0.75$ ).

\subsection{Comparison of BOLD Parameters in MS and controls groups}

Paired $t$ test showed that the $\mathrm{R}_{2}{ }^{*}$ value was lower in cortex than that in medulla. (Table 2). Independent sample t test showed that the cortical $\mathrm{R}_{2}{ }^{*}$ values were not significantly different between the two groups. Both $\mathrm{MR}_{2}{ }^{*}$ and MCR values were significantly lower in patients with MS compared with controls (Table 3 and Fig. 2).

\subsection{ROC curve analysis of BOLD Parameters}

The ROC analysis showed that the area under the curve (AUC) was 0.814 for $\mathrm{MR}_{2}{ }^{*}$, and 0.835 for $\mathrm{MCR}$ which were both higher than that of eGFR. By calculating the Youden index, optimal diagnostic threshold of $\mathrm{MR}_{2}{ }^{*}$ was $25.104 \mathrm{~Hz}$, and the sensitivity and specificity were $66.7 \%$ and $87.5 \%$, respectively. Similarity, 
optimal diagnostic threshold of MCR was 1.380 and the sensitivity and specificity were $75 \%$ and $83.3 \%$ (Table 4 and Fig. 3).

\subsection{The correlation of BOLD Parameters with eGFR}

The relationship of BOLD parameters and eGFR was analyzed by the Pearson correlation analysis. It showed that there was no statistical correlation between $\mathrm{CR}_{2}{ }^{*}$ and eGFR. However, $\mathrm{MR}_{2}{ }^{*}$ showed statistical positive correlation with eGFR $(r=0.417, P=0.003)$. Similarity, MCR also showed statistical positive correlation with eGFR $(r=0.466, P<0.001)$ (Fig. 4)

\section{Discussion}

In this study, BOLD-MRI was used to evaluate the renal oxygenation in two groups noninvasively. Intraclass correlation coefficient showed that there was favorable interobserver consistency of $R_{2}{ }^{*}$. As shown in table1, average eGFR in MS group was significantly lower than that of controls groups ( $88.35 \pm 20.19$ vs $100.26 \pm 17.36$ ), suggesting that MS group was in early renal impairment. The result also showed that $\mathrm{MR}_{2}{ }^{*}$ and MCR of MS group were significantly decreased when compared with those of the control groups. The area under the curve yielded a cut-off value of 0.814 , with sensitivity of $66.7 \%$ and specificity of $87.5 \%$ for $\mathrm{MR}_{2}{ }^{*}$. Similarity, the area under the curve yielded a cut-off value of 0.835 , with sensitivity of $75 \%$ and specificity of $83.3 \%$ for $\mathrm{MR}_{2}{ }^{*}$. Above results showed that $\mathrm{MR}_{2}{ }^{*}$ and $\mathrm{MCR}$, which were both better than eGFR, were potential imaging biomarkers for prediction of early renal impairment .

The $\mathrm{MR}_{2}{ }^{*}$ was significantly higher than $\mathrm{CR}_{2}{ }^{*}$ in both MS group and control groups, which was consistent with previous reports ${ }^{[20]}$. We considered the result was an effect of different renal regional blood distribution. It is known that human kidneys receive approximately $25 \%$ of the cardiac output and renal blood flow reaches about $400 \mathrm{ml} / 100 \mathrm{~g}$. The medulla accounts for about $10 \%$ blood flow perfusion, which is markedly less than that of cortex. Thus, $R_{2}{ }^{*}$ in the medulla is significantly higher than that of the cortex, suggesting that medullary region is relative hypoxia under physiological steady state conditions $^{[21-23]}$.

The $\mathrm{CR}_{2}{ }^{*}$ was not significantly different between MS group and control group. The results showed that oxygenation in cortex was not obviously affected by MS in the early stage of renal impairment, which may be a result of the compensatory effect of kidney. Renal oxygenation is based on a balance between oxygen supply and consumption. The oxygen supply to the cortex is well in excess of the oxygen demand under physiological steady state conditions ${ }^{[24]}$. Therefore, we speculated that oxygen was oversupplied to cortex and cortex was not in a state of hypoxia in the early stage of renal impairment. Beside, oxygen dissociation curve may be another factor accounting for the result. As it known to all, the partial pressure of oxygen is approximately $50 \mathrm{mmHg}$ in renal cortex, and approximately $10 \mathrm{mmHg}$ in renal medulla under physiological steady state conditions. The oxygen dissociation curve becomes steeper when the partial 
pressure of oxygen is less than $26.6 \mathrm{mmHg}$ and becomes flat when the partial pressure of oxygen is more than $60 \mathrm{mmHg}^{[25]}$. This indicated that small changes of oxygen partial pressure in renal cortex do not lead to evident changes of oxygenation in cortex.

Our results showed that both $\mathrm{MR}_{2}{ }^{*}$ and $\mathrm{MCR}$ measurements of MS group were significantly decreased when compared with those of control group, which was not consistent with another BOLD-MRI study of MS in animal models ${ }^{[26]}$. We believe the results could be explained in part by the different stages of renal impairment that were investigated. In the pig BOLD-MRI study, the eGFR in MS group was significantly higher than that of control groups, suggesting kidney exhibited glomerular hyperfiltration after 16 weeks of diet-induced MS. As a result, mitochondria were overwhelmed by substrate excess, leading to inefficient energy production and thereby medulla hypoxia ${ }^{[26]}$. Similarity, human kidney also exhibits glomerular hyperfiltration during early stage of $\mathrm{MS}^{[27]}$, which will theoretically lead to mitochondrial dysfunction and thereby medulla hypoxia. However, eGFR in MS group was significantly lower than that of control group in our study. Reduced glomerular filtration rate attenuated active absorption of $\mathrm{NaCl}$ in the medulla proximal tubule, which have an apparent contribution to the reduced oxygen consumption and obvious increase oxygenation in renal medulla ${ }^{[25]}$. Similar results in medulla also has been documented by BOLD-MRI in the form of CKD. In a CKD study conducted by Yuelang Zhang showed that the $\mathrm{MR}_{2}{ }^{*}$ in the control group, mild renal impairment group and moderate to severe renal impairment group gradually decreased, indicating less renal hypoxia with more severe renal damage ${ }^{[21]}$.The results from this CKD study lends support to our findings of increase oxygenation in medullary with MS, possibly due to the reduced tubular sodium reabsorption and therefore lead to reduced oxygen consumption.

In addition to the alteration of tubular reabsorption, BOLD-MRI techniques may be another possible reason that account for the apparent increase oxygenation in renal medullary in MS group. BOLD-MRI technique indirectly measures medullary oxygenation by measuring changes of regional oxygen content in the adjacent capillaries. $R_{2}{ }^{*}$ value is a measurement of endogenous capillary deoxyhemoglobin concentration, which is thought to be in equilibrium with that of the surrounding tissue under normal circumstances. However, MS could induce tubulointerstitial injury ${ }^{[28]}$. The renal tubulointerstitial fibrosis interfere with oxygen diffusion from the capillary to the surrounding medullary tissue, which lead to nonequilibrium between capillary and surrounding tissue oxygenation. Therefore, the $\mathrm{MR}_{2}{ }^{*}$ value, which is a measurement of capillary deoxyhemoglobin concentration, may be decreased because of the reduced oxygen extraction from the capillaries and ultimately lead to "increase" in medullary oxygenation ${ }^{[29]}$.

In all participants, there was no correlation between $\mathrm{CR}_{2}{ }^{*}$ and eGFR, while there was a significant positive correlation between the $\mathrm{MR}_{2}{ }^{*}$ and eGFR. This might be the result of their different sensitivity to the change of oxygen status. As mentioned above, the oxygen dissociation curve becomes steeper when oxygen pressure is less than $26.6 \mathrm{mmHg}$ and becomes flat when oxygen pressure is more than $60 \mathrm{mmHg}$. This indicated that small changes in medullary oxygen partial pressure will lead to a huge change of $\mathrm{MR}_{2}{ }^{*}$. 
The limitations of this study were as follows: (1) The sample size was relatively small and all participants were male. However, Despite the small number of subjects, there was a significant difference in the $\mathrm{MR}_{2}{ }^{*}$ between MS group and control group; (2) Renal blood flow is affected by different phases of the cardiac cycle and no information was acquired on renal perfusion in our study. We therefore can not determine whether changes in renal oxygenation were the result of alterations in renal blood flow. Nevertheless, Textor has shown that renal oxygenation is largely independent of renal blood flow, suggesting that renal blood flow did not have a huge influence on the renal $R_{2}{ }^{*}$ value ${ }^{[30]}$.

\section{Conclusion}

In conclusion, decreased $\mathrm{MR}_{2}{ }^{*}$ and $\mathrm{MCR}$ in male patients with MS were detected when compared with those in controls. The etiology for the increased oxygenation in MS is not clear. We speculate that it may be related to an alteration in oxygen consumption and possibly to factors inherent to BOLD-MRI techniques. Besides, $\mathrm{MR}_{2}{ }^{*}$ and MCR were potential imaging biomarkers for prediction of early renal impairment in male MS.

\section{Declarations}

\section{Ethics approval and consent to participate}

The protocol of this study was discussed, approved and recorded by the ethics committee of The Third Affiliated Hospital of Southern Medical University.

\section{Consent for publication and competing interests}

Written informed consent for publication was obtained from all participants and the authors declare that they have no competing interests.

\section{Availability of data and material}

The data used or analyzed during the current study are available from the corresponding author on reasonable request.

\section{Funding}

This study was supported by The Science and Technology Planning Project Of TianHe District, Guangzhou City (NO:201704KW011) and supported by National Science Foundation for Distinguished Young Scholars (NO:81801653). 


\section{Authors' Contributions}

YongQiang Li and XiaoDong Zhang conceived and designed the study. Chijian Li and Shisi Li performed the trial and wrote the paper. YiHao guo provided technical support.Shiyi Liang, Yuxiang Huang, Ge Qian and Wecheng $X v$ reviewed and edited the manuscript. All authors read and approved the manuscript.

\section{Acknowledgements}

I would like to express my gratitude to all those who helped me during the writing of this thesis. My deepest gratitude goes first and foremost to Professor Yongqiang Li, my supervisor, for his constant encouragement and guidance. He has walked me through all the stages of the writing of this thesis. Without his consistent and illuminating instruction, this thesis could not have reached its present form. Second, my thanks would go to my family for their great confidence in me all through these years. I also owe my sincere gratitude to my friends and my fellow classmates who gave me their help and time in listening to me and helping me work out my problems during the difficult course of the thesis.

\section{Trial registration}

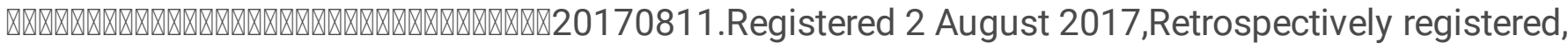
http://www.nysy.com.cn/cn/xkrc/xsjl11/

\section{References}

1. Aguilar M., Bhuket T. \& Torres S. et al., "Prevalence of the metabolic syndrome in the United States, 2003-2012.," JAMA, Vol.313, No.19(2015), pp.1973-1974.

2. Coresh J., Selvin E. \& Stevens L. A. et al., "Prevalence of chronic kidney disease in the United States.," JAMA, Vol.298, No.17(2007), pp.2038-2047.

3. Chen J., Muntner P. \& Hamm L. L. et al., "The metabolic syndrome and chronic kidney disease in U.S. adults.," Ann Intern Med, Vol.140, No.3(2004), pp.167-174.

4. Xu H., Li X. \& Adams H. et al., "Etiology of Metabolic Syndrome and Dietary Intervention.," Int J Mol Sci, Vol.20, No.1(2018).

5. Zhang X. \& Lerman L. O., "The metabolic syndrome and chronic kidney disease.," Trans/ Res, Vol.183(2017), pp.14-25.

6. Fine L. G. \& Norman J. T., "Chronic hypoxia as a mechanism of progression of chronic kidney diseases: from hypothesis to novel therapeutics.," Kidney Int, Vol.74, No.7(2008), pp.867-872.

7. Nangaku M., "Hypoxia and tubulointerstitial injury: a final common pathway to end-stage renal failure.," Nephron Exp Nephrol, Vol.98, No.1(2004), pp.e8-e12. 
8. Neugarten J. \& Golestaneh L., "Blood oxygenation level-dependent MRI for assessment of renal oxygenation.," Int J Nephrol Renovasc Dis, Vol.7(2014), pp.421-435.

9. Ebrahimi B., Textor S. C. \& Lerman L. O., "Renal relevant radiology: renal functional magnetic resonance imaging.," Clin J Am Soc Nephrol, Vol.9, No.2(2014), pp.395-405.

10. Wang Y. T., Li Y. C. \& Yin L. L. et al., "Functional assessment of transplanted kidneys with magnetic resonance imaging.," World J Radiol, Vol.7, No.10(2015), pp.343-349.

11. Alford S. K., Sadowski E. A. \& Unal O. et al., "Detection of acute renal ischemia in swine using blood oxygen level-dependent magnetic resonance imaging.," J Magn Reson Imaging, Vol.22, No.3(2005), pp.347-353.

12. Djamali A., Sadowski E. A. \& Muehrer R. J. et al., "BOLD-MRI assessment of intrarenal oxygenation and oxidative stress in patients with chronic kidney allograft dysfunction., Am J Physiol Renal Physiol, Vol.292, No.2(2007), pp.F513-F522.

13. Hofmann L., Simon-Zoula S. \& Nowak A. et al., "BOLD-MRI for the assessment of renal oxygenation in humans: acute effect of nephrotoxic xenobiotics.," Kidney Int, Vol.70, No.1(2006), pp.144-150.

14. Ries M., Basseau F. \& Tyndal B. et al., "Renal diffusion and BOLD MRI in experimental diabetic nephropathy. Blood oxygen level-dependent.," J Magn Reson Imaging, Vol.17, No.1(2003), pp.104113.

15. Eirin A., Woollard J. R. \& Ferguson C. M. et al., "The metabolic syndrome induces early changes in the swine renal medullary mitochondria.," Transl Res, Vol.184(2017), pp.45-56.

16. Jia W., Weng J. \& Zhu D. et al., "Standards of medical care for type 2 diabetes in China 2019.," Diabetes Metab Res Rev, Vol.35, No.6(2019), p.e3158.

17. Ma Y. C., Zuo L. \& Chen J. H. et al., "Modified glomerular filtration rate estimating equation for Chinese patients with chronic kidney disease.," J Am Soc Nephrol, Vol.17, No.10(2006), pp.29372944.

18. Matthews D. R., Hosker J. P. \& Rudenski A. S. et al., "Homeostasis model assessment: insulin resistance and beta-cell function from fasting plasma glucose and insulin concentrations in man.," Diabetologia, Vol.28, No.7(1985), pp.412-419.

19. Pedersen M., Dissing T. H. \& Morkenborg J. et al., "Validation of quantitative BOLD MRI measurements in kidney: application to unilateral ureteral obstruction.," Kidney Int, Vol.67, No.6(2005), pp.2305-2312.

20. Gloviczki M. L., Glockner J. \& Gomez S. I. et al., "Comparison of 1.5 and 3 T BOLD MR to study oxygenation of kidney cortex and medulla in human renovascular disease.," Invest Radiol, Vol.44, No.9(2009), pp.566-571.

21. Li C., Liu H. \& Li X. et al., "Application of BOLD-MRI in the classification of renal function in chronic kidney disease.," Abdom Radiol (NY), Vol.44, No.2(2019), pp.604-611.

22. Yin W. J., Liu F. \& Li X. M. et al., "Noninvasive evaluation of renal oxygenation in diabetic nephropathy by BOLD-MRI.," Eur J Radiol, Vol.81, No.7(2012), pp.1426-1431. 
23. , "A preliminary study of blood-oxygen-level-dependent MRI in patients with chronic kidney disease. Magn Reson Imaging, 2012,".

24. Hansell P., Welch W. J. \& Blantz R. C. et al., "Determinants of kidney oxygen consumption and their relationship to tissue oxygen tension in diabetes and hypertension.," Clin Exp Pharmacol Physiol, Vol.40, No.2(2013), pp.123-137.

25. Brezis M. \& Rosen S., "Hypoxia of the renal medulla--its implications for disease.," N Engl J Med, Vol.332, No.10(1995), pp.647-655.

26. Eirin A., Woollard J. R. \& Ferguson C. M. et al., "The metabolic syndrome induces early changes in the swine renal medullary mitochondria.," Transl Res, Vol.184(2017), pp.45-56.

27. M M., L P. \& G B. et al., "Glomerular hyperfiltration and metabolic syndrome: results from the FlrenzeBAgno A Ripoli (FIBAR) Study.," Acta diabetologica, Vol.46, No.3(2009), pp.191-196.

28. Wang C., Blough E. R. \& Arvapalli R. et al., "Metabolic syndrome-induced tubulointerstitial injury: role of oxidative stress and preventive effects of acetaminophen.," Free Radic Biol Med, Vol.65(2013), pp.1417-1426.

29. Wang Z. J., Kumar R. \& Banerjee S. et al., "Blood oxygen level-dependent (BOLD) MRI of diabetic nephropathy: preliminary experience.," J Magn Reson Imaging, Vol.33, No.3(2011), pp.655-660.

30. Gloviczki M. L., Glockner J. F. \& Crane J. A. et al., "Blood oxygen level-dependent magnetic resonance imaging identifies cortical hypoxia in severe renovascular disease.," Hypertension, Vol.58, No.6(2011), pp.1066-1072.

\section{Tables}

Table 1. Baseline characteristics of MS and controls enrolled in the study. 


\begin{tabular}{|c|c|c|c|}
\hline & $M S \bigotimes n=24 \rrbracket$ & Controls $\triangle n=24 \rrbracket$ & $\mathrm{P}$ \\
\hline Age $\bigotimes$ years $\rrbracket$ & $37.38 \pm 9.05$ & $34.42 \pm 7.75$ & 0.230 \\
\hline Weight $\llbracket \mathrm{kg} \rrbracket$ & $89.44 \pm 13.58$ & $65.93 \pm 8.75$ & $<0.001$ \\
\hline Height $\rrbracket m \rrbracket$ & $1.71 \pm 0.56$ & $1.70 \pm 0.69$ & 0.599 \\
\hline $\mathrm{BMI} \triangle \mathrm{kg} / \mathrm{m}^{2} \mathrm{\square}$ & $30.43 \pm 4.33$ & $22.67 \pm 2.67$ & $<0.001$ \\
\hline $\mathrm{SBP} \otimes \mathrm{mmHg} \rrbracket$ & $141 \pm 11.10$ & $124 \pm 9.19$ & $<0.001$ \\
\hline $\mathrm{DBP} \rrbracket \mathrm{mmHg} \rrbracket$ & $86.33 \pm 8.84$ & $73.67 \pm 8.32$ & $<0.001$ \\
\hline Waist circumference $\llbracket \mathrm{cm} \rrbracket$ & $105.34 \pm 10.10$ & $83.45 \pm 6.30$ & $<0.001$ \\
\hline Serum creatitine\uml/L】 & $94.46 \pm 17.36$ & $86.75 \pm 12.53$ & 0.085 \\
\hline $\mathrm{eGFR} \rrbracket \mathrm{mL} / \mathrm{min} / 1.73 \mathrm{~m}^{2} \square$ & $88.35 \pm 20.19$ & $100.26 \pm 17.36$ & 0.034 \\
\hline Fast insulin $\rrbracket \mathrm{uU} / \mathrm{ml} \rrbracket$ & $20.44 \pm 9.78$ & $8.48 \pm 4.11$ & $<0.001$ \\
\hline HOMA-IR index & $1.88 \pm 0.34$ & $1.13 \pm 0.34$ & $<0.01$ \\
\hline Fast blood glucose $\ \mathrm{mmol} / \mathrm{L} \rrbracket$ & $6.11 \pm 1.39$ & $4.90 \pm 0.46$ & $<0.001$ \\
\hline Serum uric acid】umol/L】 & $420.75 \pm 102.51$ & $390.96 \pm 92.17$ & 0.295 \\
\hline Total cholesterol $囚 \mathrm{mmol} / \mathrm{LQ}$ & $4.65 \pm 1.15$ & $4.71 \pm 0.85$ & 0.839 \\
\hline Triglyceride $\bigotimes \mathrm{mmol} / \mathrm{L} \bigotimes$ & $2.61 \pm 1.48$ & $1.19 \pm 0.24$ & $<0.001$ \\
\hline Low density lipoprotein $₫ \mathrm{mmol} / \mathrm{L} \bigotimes$ & $2.21 \pm 0.99$ & $2.62 \pm 0.20$ & 0.158 \\
\hline High density lipoprotein $₫ \mathrm{mmol} / \mathrm{L} \bigotimes$ & $1.22 \pm 0.30$ & $1.52 \pm 0.38$ & 0.004 \\
\hline Aspartate aminotransferase(U/L) & $43.00 \pm 25.65$ & $23 \pm 16.34$ & 0.002 \\
\hline Alanine aminotransferase $(\mathrm{U} / \mathrm{L})$ & $28.50 \pm 13.33$ & $21.83 \pm 8.79$ & 0.047 \\
\hline Leukocyte(x10^9/L) & $7.11 \pm 2.68$ & $6.08 \pm 0.83$ & 0.083 \\
\hline 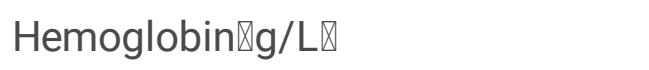 & $154.25 \pm 11.23$ & $150.83 \pm 11.79$ & 0.309 \\
\hline
\end{tabular}

Table 2. Comparison of $\mathrm{R}_{2}{ }^{*}$ between cortex and medulla.

\begin{tabular}{|lllll|}
\hline & Cortex $\mathrm{R}_{2}{ }^{*}$ & Medulla $\mathrm{R}_{2}{ }^{*}$ & $\mathrm{t}$ & $\mathrm{p}$ \\
\hline $\mathrm{MS} \rrbracket \mathrm{n}=24 \rrbracket$ & $19.04 \pm 1.64$ & $24.39 \pm 2.18$ & -9.750 & $<0.001$ \\
\hline Controls $₫ \mathrm{n}=24 \rrbracket$ & $18.54 \pm 1.75$ & $28.21 \pm 4.70$ & -10.936 & $<0.001$ \\
\hline
\end{tabular}


Table 3. Comparison of BOLD Parameters in MS and Control group.

\begin{tabular}{|lllll|}
\hline & $M S \triangle n=24 \rrbracket$ & Controls $₫ n=24 \rrbracket$ & $t$ & $P$ \\
\hline Cortex $R_{2}{ }^{*}$ & $19.04 \pm 1.64$ & $18.54 \pm 1.75$ & 1.017 & 0.315 \\
Medulla $R_{2}{ }^{*}$ & $24.39 \pm 2.18$ & $28.21 \pm 4.70$ & -3.612 & 0.001 \\
MCR & $1.29 \pm 0.14$ & $1.53 \pm 0.28$ & -3.836 & $<0.001$ \\
\hline
\end{tabular}

Table 4: Diagnostic efficiency of BOLD parameters and clinical index of renal function

\begin{tabular}{|lllllll|}
\hline & AUC & Sensitivity & Specificity & Cut-off point & $95 \% \mathrm{Cl}$ & $\mathrm{P}$ \\
\hline Cortex $\mathrm{R}_{2}{ }^{*}$ & 0.392 & 1.000 & 0.042 & 22.180 & $0.229-0.556$ & 0.201 \\
\hline Medulla $\mathrm{R}_{2}{ }^{*}$ & 0.814 & 0.667 & 0.875 & 25.104 & $0.690-0.938$ & $<0.001$ \\
\hline MCR & 0.835 & 0.750 & 0.833 & 1.380 & $0.715-0.955$ & $<0.001$ \\
SCR & 0.360 & 0.125 & 1.00 & 70.500 & $0.198-0.522$ & 0.097 \\
eGFR & 0.682 & 0.667 & 0.750 & 93.093 & $0.526-0.839$ & 0.030 \\
\hline
\end{tabular}

\section{Figures}

A

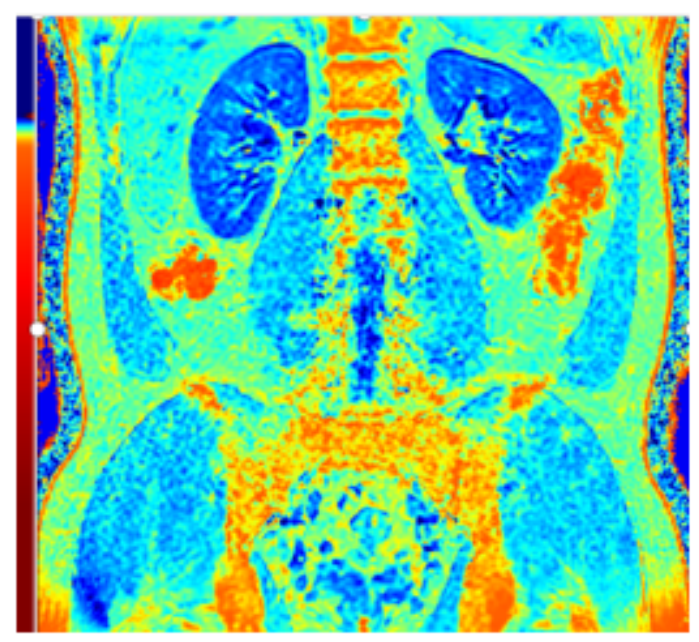

B

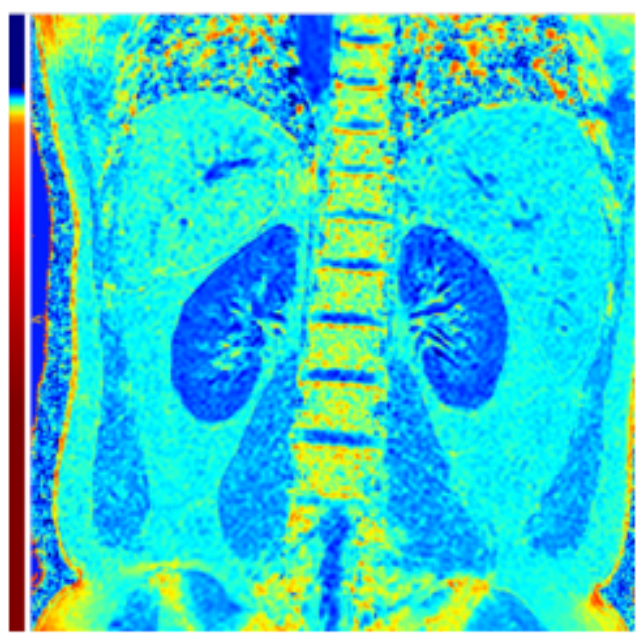

Figure 1 
R2* map in coronal plane of the kidneys in a 36-year-old healthy volunteer. B:R2* map in coronal plane of the kidneys in a 37-year-old MS patient.
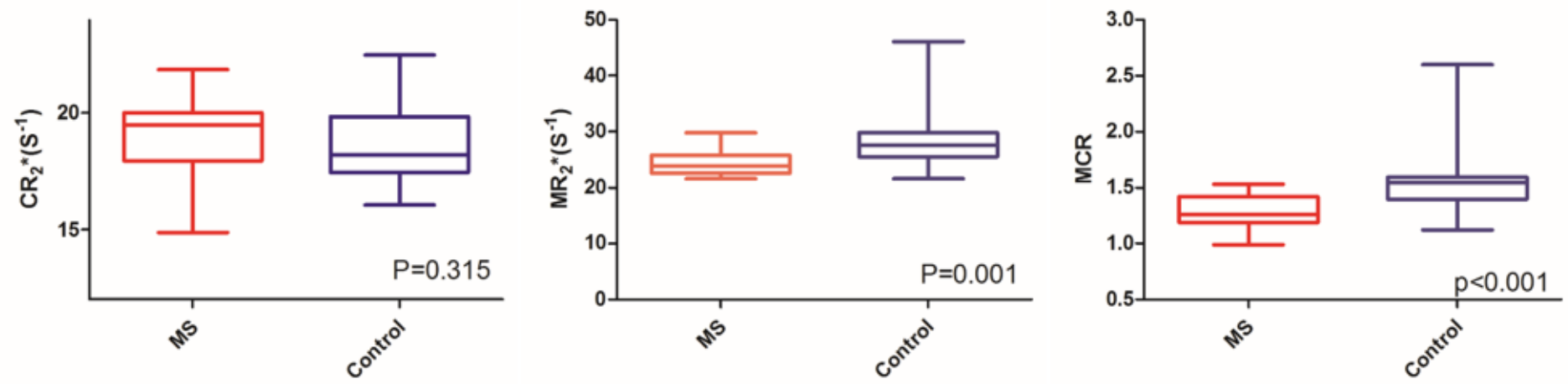

Figure 2

Comparison of BOLD Parameters in MS and Control group. 
ROC Curve

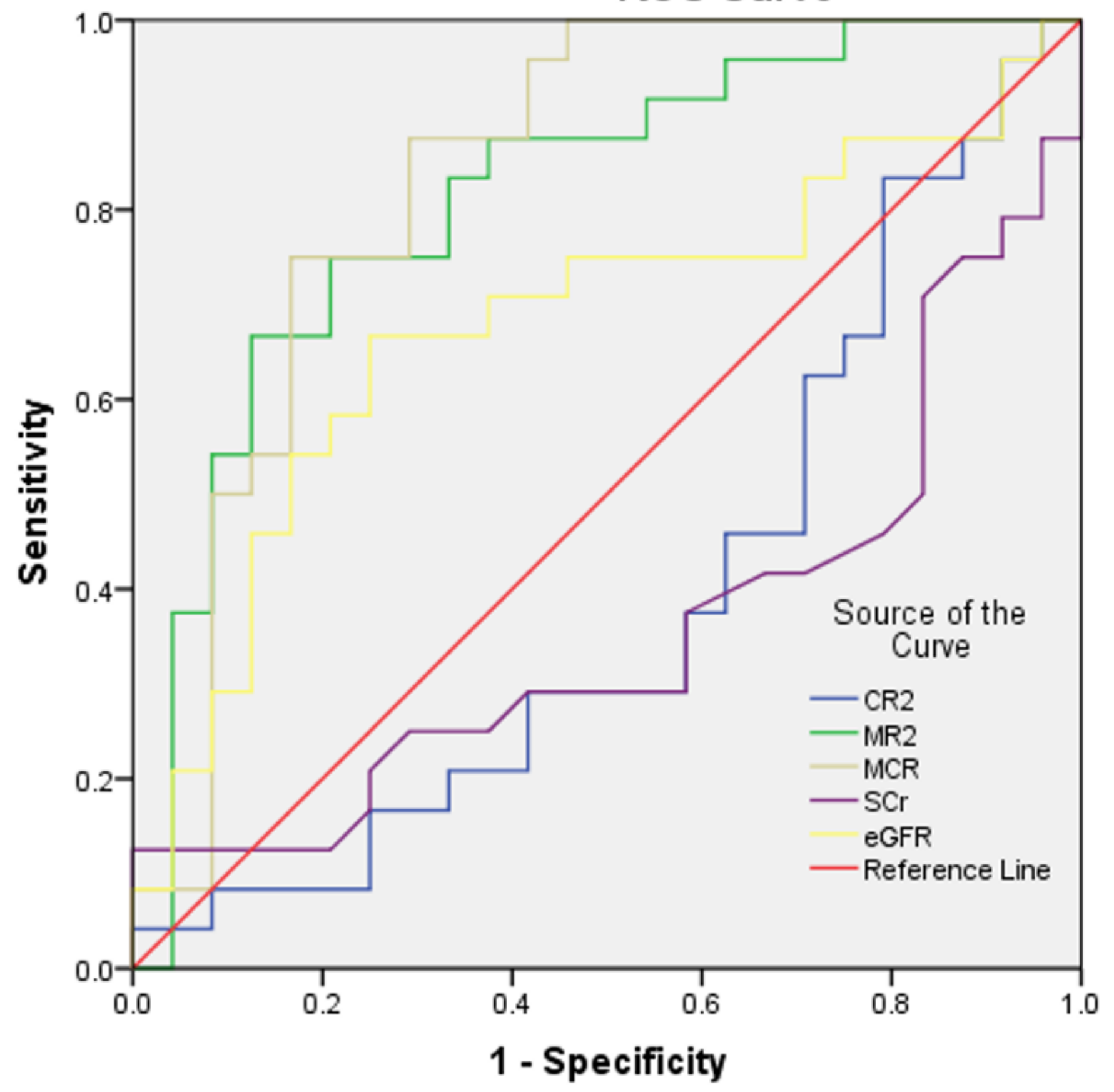

Diagonal segments are produced by ties.

Figure 3

Diagnostic efficiency of BOLD Parameters and clinical index of renal function 

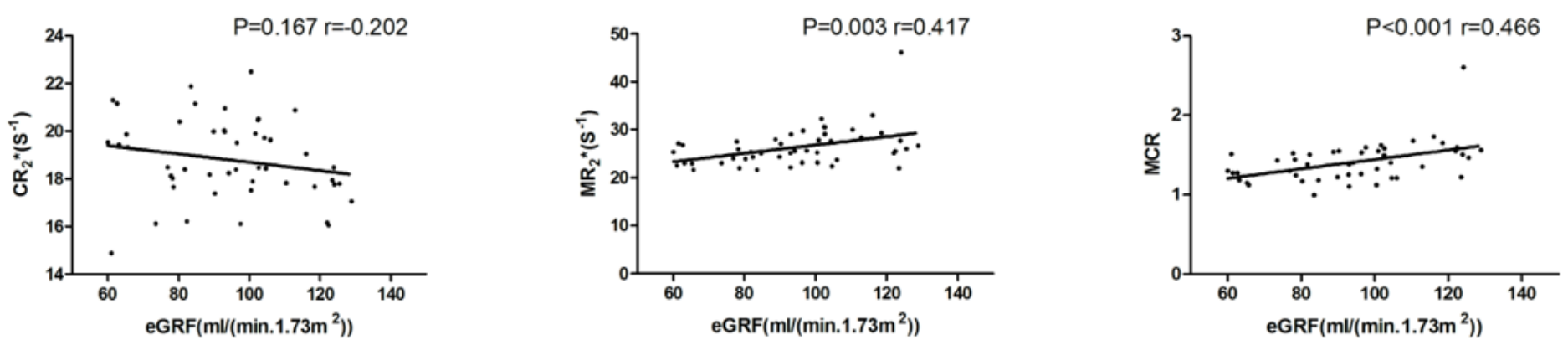

\section{Figure 4}

The correlation of BOLD Parameters with eGFR. 